TRANSACTIONS OF THE

AMERICAN MATHEMATICAL SOCIETY

Volume 355, Number 8 , Pages $3227-3239$

S 0002-9947(03)03287-2

Article electronically published on March 12, 2003

\title{
ON MEASURES OF MAXIMAL AND FULL DIMENSION FOR POLYNOMIAL AUTOMORPHISMS OF $\mathbb{C}^{2}$
}

\author{
CHRISTIAN WOLF
}

\begin{abstract}
For a hyperbolic polynomial automorphism of $\mathbb{C}^{2}$, we show the existence of a measure of maximal dimension and identify the conditions under which a measure of full dimension exists.
\end{abstract}

\section{INTRODUCTION}

Let $g$ be a hyperbolic polynomial automorphism of $\mathbb{C}^{2}$. For $A \subset \mathbb{C}^{2}$ we denote by $\operatorname{dim}_{H} A$ the Hausdorff dimension of $A$. Let $\nu$ be an invariant Borel probability measure. We define the Hausdorff dimension of $\nu$ by

$$
\operatorname{dim}_{H}(\nu)=\inf \left\{\operatorname{dim}_{H} A: \nu(A)=1\right\} .
$$

We define the quantity $\delta(g)$ by

$$
\delta(g)=\sup \left\{\operatorname{dim}_{H}(\nu)\right\}
$$

where the supremum is taken over all ergodic invariant Borel probability measures with positive entropy 1 This quantity was introduced by Denker and Urbanski [DU] in the context of rational maps on the Riemann sphere. They called it the dynamical dimension of the map.

It is easy to see that the support of each measure considered in (2) is contained in the Julia set $J$ (see Section 2 for the definition). We denote by $M\left(J,\left.g\right|_{J}\right)$ the set of all ergodic invariant Borel probability measures supported on $J$.

If a measure $\nu \in M\left(J,\left.g\right|_{J}\right)$ attains the supremum in (2), that is,

$$
\operatorname{dim}_{H}(\nu)=\delta(g),
$$

we say that $\nu$ is a measure of maximal dimension for $g$.

McCluskey and Manning [MM] gave a heuristic argument for the existence of a measure of maximal dimension in the case of Axiom A surface diffeomorphisms. However, it was not known until this paper whether this argument can be extended to a rigorous proof (see the remarks after Theorem 5.2 for more details). 2

Received by the editors July 30, 2001 and, in revised form, December 11, 2002.

2000 Mathematics Subject Classification. Primary 37C45, 37D35, 32H50; Secondary 37D20, $37 \mathrm{FXX}$.

The author was supported by a research fellowship of the Deutsche Forschungsgemeinschaft (DFG).

${ }^{1}$ Using Young's formula $[\mathrm{Y}]$ and results by L. Barreira and the author [BW1 it is possible to show that the value of $\delta(f)$ remains the same when the supremum is taken over all invariant Borel probability measures.

${ }^{2}$ While this paper was in the process of publishing, L. Barreira and the author [BW2] proved the existence of a measure of maximal dimension for Axiom A surface diffeomorphisms.

(C)2003 American Mathematical Society 
In this paper we study the existence of a measure of maximal dimension for hyperbolic polynomial automorphisms of $\mathbb{C}^{2}$. Our main result is the following (see Theorems 5.1, 5.2 and Corollary [5.4).

Theorem 1.1. Let $g$ be a hyperbolic polynomial automorphism of $\mathbb{C}^{2}$. Then there exists a measure of maximal dimension for $g$. The set of measures of maximal dimension is finite.

The proof of this theorem uses the theory of thermodynamic formalism. Other crucial ingredients are Young's formula $\bar{Y}$, which relates the dimension of a measure to entropy and Lyapunov exponents, and dimension-theoretic results pertaining to the dynamics of polynomial automorphisms of $\mathbb{C}^{2}$ (see Wo1, Wo2 ). The key idea is to extract a one-parameter family of potentials and to consider the corresponding family of equilibrium measures. We show that a measure of maximal dimension necessarily belongs to this family of equilibrium measures. Furthermore, if a measure $\nu$ maximizes Hausdorff dimension among these equilibrium measures, then $\nu$ is a measure of maximal dimension.

We say that $\nu \in M\left(J,\left.g\right|_{J}\right)$ is a measure of full dimension if

$$
\operatorname{dim}_{H}(\nu)=\operatorname{dim}_{H} J .
$$

We prove in Corollary 3.5 that there exists at most one measure of full dimension. The next result gives a classification for the existence of a measure of full dimension (see Theorems 4.1 and 4.2).

Theorem 1.2. Let $g$ be a hyperbolic polynomial automorphism of $\mathbb{C}^{2}$. Then

i) If $g$ is volume-preserving, then there exists a unique measure of full dimension for $g$.

ii) If $g$ does not preserve volume, and if $g$ admits a measure of full dimension, then this measure is the unique measure of maximal entropy.

In the volume-preserving case the existence of a measure of full dimension has already been shown by Friedland and Ochs [FO]. We provide an alternative proof for this result in Theorem 4.1

Theorem 1.2 indicates that in the case of non-volume-preserving maps, the existence of a measure of full dimension seems to be a rare phenomenon. Indeed, we show in Theorem 4.2 that the existence of such a measure is equivalent to the fact that the multipliers of all saddle points are of the same modulus (see equation (28) for the precise statement). If this condition would be satisfied, then the parameter defining the map $g$ would provide solutions of a countable infinite set of algebraic equations. From this point of view, such an example seems very likely to not exist.

In Section 4 we observe that there exists a dense open subset of hyperbolic parameter space for which no measure of full dimension exists. This implies that

$$
\delta(g)<\operatorname{dim}_{H} J
$$

holds for these parameters.

In the last part of this paper, we analyze the dependence of $\delta(g)$ on the parameter of the mapping. More precisely, we prove the following result (see Theorems 6.1 and 6.2.

Theorem 1.3. Let $\lambda \mapsto g_{\lambda}$ be a holomorphic family of hyperbolic polynomial automorphisms of $\mathbb{C}^{2}$. Then $\lambda \mapsto \delta\left(g_{\lambda}\right)$ is continuous and plurisubharmonic. 
This paper is organized as follows. In Section 2 we present the basic definitions and notation, and also list the standing assumptions of the paper. In Section 3 we introduce elements from dimension theory for hyperbolic polynomial automorphisms of $\mathbb{C}^{2}$ and provide the tools for the analysis of the existence of measures of maximal and full dimension. Section 4 is devoted to the analysis of the existence of a measure of full dimension. The existence of a measure of maximal dimension is proved in Section 5. Finally, we study in Section 6 the dependence of $\delta(g)$ on parameters.

It would be interesting to understand whether, or at least under which conditions, a uniqueness result for the measure of maximal dimension holds. A partial answer to this question is given in Corollary 3.5 where a uniqueness result is shown in the case when a measure of full dimension exists.

\section{Notation and Preliminaries}

Let $g$ be a polynomial automorphism of $\mathbb{C}^{2}$. We denote by $\operatorname{deg}(g)$ the maximum of the polynomial degree of the components of $g$. The dynamical degree of $g$ is defined by

$$
d=\lim _{n \rightarrow \infty}\left(\operatorname{deg}\left(g^{n}\right)\right)^{1 / n}
$$

(see $\overline{B S 2},[\overline{F M}]$ ). We are interested in nontrivial dynamics, which occurs if and only if $d>1$. Friedland and Milnor showed in $[\mathrm{FM}$ that every polynomial automorphism of $\mathbb{C}^{2}$ with nontrivial dynamics is conjugate to a mapping of the form $g=g_{1} \circ \ldots \circ g_{m}$, where each $g_{i}$ is a generalized Hénon mapping. This means that $g_{i}$ has the form

$$
g_{i}(z, w)=\left(w, P_{i}(w)+a_{i} z\right),
$$

where $P_{i}$ is a complex polynomial of degree $d_{i} \geq 2$ and $a_{i}$ is a nonzero complex number. The dynamical degree $d$ of such a map $g$ is equal to $d_{1} \cdot \ldots \cdot d_{m}$ and therefore coincides with the polynomial degree of $g$.

For $g$ we define $K^{ \pm}$as the set of points in $\mathbb{C}^{2}$ with bounded forward/backward orbits, $K=K^{+} \cap K^{-}, J^{ \pm}=\partial K^{ \pm}$and $J=J^{+} \cap J^{-}$. We refer to $J^{ \pm}$as the positive/negative Julia set of $g$ and call $J$ the Julia set of $g$. The sets $K$ and $J$ are compact.

Note that the function $a=\operatorname{det} D g$ is constant in $\mathbb{C}^{2}$. Therefore, we can restrict our considerations to the volume-decreasing case $(|a|<1)$, and to the volumepreserving case $(|a|=1)$, because in the volume-increasing case $(|a|>1)$, we can consider $g^{-1}$.

As pointed out in the introduction we assume in this paper that $g$ is a hyperbolic mapping. This means that there exists a continuous invariant splitting $T_{J} \mathbb{C}^{2}=E^{u} \oplus E^{s}$ such that $\left.D g\right|_{E^{u}}$ is uniformly expanding and $\left.D g\right|_{E^{s}}$ is uniformly contracting. Hyperbolicity implies that we can associate with each point $p \in J$ its local unstable/stable manifold $W_{\epsilon}^{u / s}(p)$. Moreover, $g$ is an Axiom A diffeomorphism (see [BS1] for more details).

Standing Assumptions. We now list several conditions which will be assumed throughout the entire paper:

i) $g$ is a polynomial automorphism of $\mathbb{C}^{2}$ with dynamical degree $d>1$;

ii) $g$ is hyperbolic;

iii) $g$ is volume-non-increasing. 
We recall that assumption iii) is actually not a restriction since we can also consider $g^{-1}$ (see above).

\section{Elements from Dimension Theory}

In this section we introduce elements from dimension theory for hyperbolic polynomial automorphisms of $\mathbb{C}^{2}$ and provide the tools for the analysis of measures of maximal and full dimension.

We start by introducing Lyapunov exponents. Let $\nu \in M\left(J,\left.g\right|_{J}\right)$. The Julia set $J$ is a hyperbolic set of index 1 (see [BS1]). Therefore, by the multiplicative ergodic theorem of Oseledec, there are Lyapunov exponents $\lambda_{\nu}^{-}<0<\lambda_{\nu}^{+}$with respect to $\nu$ (see e.g. $[\mathrm{KH}]$ ). In particular, $\nu$ is a hyperbolic measure. We define the quantity

$$
\Lambda(\nu)=\lim _{n \rightarrow \infty} \frac{1}{n} \int_{J} \log \left\|D g^{n}\right\| d \nu .
$$

Similar to what was done for the measure of maximal entropy in BS3, the positive Lyapunov exponent $\lambda_{\nu}^{+}$coincides with $\Lambda(\nu)$. Since $g$ has constant Jacobian determinant $a$, the negative Lyapunov exponent $\lambda_{\nu}^{-}$is given by $-\Lambda(\nu)+\log |a|$. In Wo1 we derived the formula

$$
\Lambda(\nu)=\left.\int_{J} \log || D g\right|_{E^{u}} \| d \nu .
$$

By Young's formula $\left[\mathbf{Y}\right.$, we have for all $\nu \in M\left(J,\left.g\right|_{J}\right)$ that

$$
\operatorname{dim}_{H}(\nu)=\frac{h_{\nu}(g)}{\Lambda(\nu)}+\frac{h_{\nu}(g)}{\Lambda(\nu)-\log |a|} .
$$

Here $h_{\nu}(g)$ denotes the measure-theoretic entropy of $g$ with respect to $\nu$.

Next we introduce topological pressure. Let $C(J, \mathbf{R})$ denote the Banach space of all continuous functions from $J$ to $\mathbf{R}$. The topological pressure of $\left.g\right|_{J}$, denoted by $P=P\left(\left.g\right|_{J},.\right)$, is a mapping from $C(J, \mathbf{R})$ to $\mathbf{R}$ (see Wa for the definition). The variational principle provides the formula

$$
P\left(\left.g\right|_{J}, \varphi\right)=\sup _{\nu \in M\left(J,\left.g\right|_{J}\right)}\left(h_{\nu}(g)+\int_{J} \varphi d \nu\right) .
$$

If a measure $\nu_{\varphi} \in M\left(J,\left.g\right|_{J}\right)$ achieves the supremum in equation (10), that is,

$$
P\left(\left.g\right|_{J}, \varphi\right)=h_{\nu_{\varphi}}(g)+\int_{J} \varphi d \nu_{\varphi},
$$

it is called an equilibrium measure of the potential $\varphi$.

The topological pressure has the following properties (see $[\mathrm{R}]$ ).

i) The topological pressure is a convex function.

ii) If $\varphi$ is a strictly negative function, then the function $t \mapsto P\left(\left.g\right|_{J}, t \varphi\right)$ is strictly decreasing.

iii) The topological pressure is a real analytic function on the subspace of Hölder continuous functions, that is, when $\alpha>0$ is fixed, then $\left.P\left(\left.g\right|_{J},.\right)\right|_{C^{\alpha}(J, \mathbf{R})}$ is a real analytic function. Note that $C^{\alpha}$ cannot be replaced by $C^{0}$. 
iv) If $\alpha>0$ and $\varphi \in C^{\alpha}(J, \mathbf{R})$, then there exists a uniquely defined equilibrium measure $\nu_{\varphi} \in M\left(J,\left.g\right|_{J}\right)$ of the potential $\varphi$. Furthermore, we have for all $\varphi, \psi \in C^{\alpha}(J, \mathbf{R})$,

$$
\left.\frac{d}{d t}\right|_{t=0} P\left(\left.g\right|_{J}, \varphi+t \psi\right)=\int_{J} \psi d \nu_{\varphi}
$$

We have $P\left(\left.g\right|_{J}, 0\right)=h_{\text {top }}\left(\left.g\right|_{J}\right)$ (see e.g. [Wa]), where $h_{\text {top }}\left(\left.g\right|_{J}\right)$ denotes the topological entropy of $\left.g\right|_{J}$. Therefore, by property iv), the equilibrium measure of the potential constant zero, $\nu_{0}$, is the unique measure of maximal entropy of $\left.g\right|_{J}$. The map $g$ has a unique measure of maximal entropy (see [BS1]); moreover, this measure is supported on $J$. We conclude that $\nu_{0}$ is the unique measure of maximal entropy of $g$. We now introduce potentials which are related to Lyapunov exponents. We define

$$
\phi^{u / s}: J \rightarrow \mathbf{R}, \quad p \mapsto \log \left\|\left.D g(p)\right|_{E_{p}^{u / s}}\right\|
$$

and the unstable/stable pressure functions

$$
P^{u / s}: \mathbf{R} \rightarrow \mathbf{R}, \quad t \mapsto P\left(\left.g\right|_{J}, \mp t \phi^{u / s}\right) .
$$

The Julia set $J$ is a hyperbolic set of index 1; thus the potentials $\mp \phi^{u / s}$ are strictly negative. Therefore, property ii) of the topological pressure implies that the functions $P^{u / s}$ are strictly decreasing.

Since $\phi^{u / s}$ are Hölder continuous (see [B]), we may conclude from property iii) of the topological pressure that $P^{u / s}$ are real analytic. Property iv) of the topological pressure implies that there exist uniquely defined equilibrium measures $\nu_{\mp t \phi^{u / s}} \in$ $M\left(J,\left.g\right|_{J}\right)$ of the potentials $\mp t \phi^{u / s}$.

We will need the following result about the relation between the unstable and stable pressure functions.

Proposition 3.1 ([फo2] $)$. $P^{u}(t)=P^{s}(t)-t \log |a|$.

Lemma 3.2. $\nu_{-t \phi^{u}}=\nu_{t \phi^{s}}$.

Proof. Let $t \geq 0$. Then

$$
\begin{aligned}
P^{s}(t) & =P^{u}(t)+t \log |a| \\
& =h_{\nu_{-t \phi^{u}}}(g)+t\left(-\left.\int \log || D g\right|_{E^{u}} \| d \nu_{-t \phi^{u}}+\log |a|\right) \\
& =h_{\nu_{-t \phi^{u}}}(g)+t\left(\lim _{n \rightarrow \infty} \frac{1}{n} \int-\log \left\|\left.D g^{n}\right|_{E^{u}}\right\|+\log \left|a^{n}\right| d \nu_{-t \phi^{u}}\right) \\
& =h_{\nu_{-t \phi^{u}}}(g)+t\left(\lim _{n \rightarrow \infty} \frac{1}{n} \int \log \left\|\left.D g^{n}\right|_{E^{s}}\right\| d \nu_{-t \phi^{u}}\right) \\
& =h_{\nu_{-t \phi^{u}}}(g)+t \int \log \left\|\left.D g\right|_{E^{s}}\right\| d \nu_{-t \phi^{u}} \\
& =h_{\nu_{-t \phi^{u}}}(g)+t \int \phi^{s} d \nu_{-t \phi^{u}} .
\end{aligned}
$$

The result follows from the uniqueness of the equilibrium measure of the potential $t \phi^{s}$.

We will use in the remainder of this paper the notation $\nu_{t}=\nu_{\mp t \phi^{u / s}}$. This notation is justified by Lemma 3.2. We also write $\Lambda(t)=\Lambda\left(\nu_{t}\right)$ and $h(t)=h_{\nu_{t}}(g)$, 
and consider $\Lambda$ and $h$ as real-valued functions of $t$. Equations (8) and (11) imply that

$$
P^{u}(t)=h(t)-t \Lambda(t)
$$

Therefore, Proposition 3.1 implies that

$$
P^{s}(t)=h(t)-t(\Lambda(t)-\log |a|) .
$$

Proposition 3.3. $\Lambda$ and $h$ are real analytic. Furthermore,

$$
\frac{d \Lambda}{d t} \leq 0
$$

If $\Lambda$ is not constant, then every zero of the derivative of $\Lambda$ is isolated, and $\Lambda$ is strictly decreasing.

Proof. Let $t_{0} \geq 0$ and let $\phi^{u}$ be as in (13). We define potentials $\varphi=-t_{0} \phi^{u}, \psi=$ $-\phi^{u}$ (here we use the notation of (12)). Therefore, application of equations (8) and (12) implies that

$$
\frac{d P^{u}}{d t}\left(t_{0}\right)=-\Lambda\left(\nu_{t_{0}}\right)=-\Lambda\left(t_{0}\right)
$$

Since $P^{u}$ is real analytic, we obtain that $\Lambda$ is also real analytic. We conclude from (16) that $h$ is also real analytic. The convexity of $P^{u}$ implies that

$$
\frac{d^{2} P^{u}}{d t^{2}} \geq 0
$$

hence

$$
\frac{d \Lambda}{d t} \leq 0
$$

Finally, if $\Lambda$ is not constant, then the uniqueness theorem for real analytic functions, applied to the derivative of $\Lambda$, implies that all zeros of the derivative of $\Lambda$ are isolated. Therefore, $\Lambda$ is strictly decreasing.

Hausdorff dimensions of the measures $\nu_{t}$. We use the notation $\Delta(t)=$ $\operatorname{dim}_{H}\left(\nu_{t}\right)$. Equation (9) yields

$$
\Delta(t)=\frac{h(t)}{\Lambda(t)}+\frac{h(t)}{\Lambda(t)-\log |a|} .
$$

Thus, $\Delta$ is also a real analytic function. Equations (16), (17) and Proposition 3.1 imply that

$$
\Delta(t)=2 t+\frac{P^{u}(t)}{\Lambda(t)}+\frac{P^{u}(t)+t \log |a|}{\Lambda(t)-\log |a|} .
$$

From an elementary calculation we obtain the following formula for the derivative of $\Delta$ :

$$
\frac{d \Delta}{d t}\left(t_{0}\right)=-\frac{\frac{d \Lambda}{d t}\left(t_{0}\right)\left[P^{u}\left(t_{0}\right)\left(\Lambda\left(t_{0}\right)-\log |a|\right)^{2}+\left(P^{u}\left(t_{0}\right)+t_{0} \log |a|\right) \Lambda\left(t_{0}\right)^{2}\right]}{\Lambda\left(t_{0}\right)^{2}\left(\Lambda\left(t_{0}\right)-\log |a|\right)^{2}} .
$$

Hausdorff dimension of $J$. The following result due to Verjovsky and Wu provides a formula for the Hausdorff dimension of the unstable/stable slice in terms of the zeros of the pressure functions. 
Theorem $3.4([\mathbb{V W}])$. Let $p \in J$. Then $t^{u / s}=\operatorname{dim}_{H} W_{\epsilon}^{u / s}(p) \cap J$ does not depend on $p \in J$. Furthermore, $t^{u / s}$ is given by the unique solution of

$$
P^{u / s}(t)=0 .
$$

Equation (25) is called the Bowen-Ruelle formula. We refer to $t^{u / s}$ as the Hausdorff dimension of the unstable/stable slice.

In [Wo1] we proved the formula

$$
\operatorname{dim}_{H} J=t^{u}+t^{s}=\sup _{\nu \in M\left(J,\left.g\right|_{J}\right)}\left(\frac{h_{\nu}(g)}{\Lambda(\nu)}\right)+\sup _{\nu \in M\left(J,\left.g\right|_{J}\right)}\left(\frac{h_{\nu}(g)}{\Lambda(\nu)-\log |a|}\right),
$$

where each of the suprema on the right-hand side of the equation is uniquely attained by the measures $\nu_{t^{u}}$ and $\nu_{t^{s}}$, respectively. Hence

$$
\operatorname{dim}_{H} J=\frac{h\left(t^{u}\right)}{\Lambda\left(t^{u}\right)}+\frac{h\left(t^{s}\right)}{\Lambda\left(t^{s}\right)-\log |a|} .
$$

Equation (27) and the uniqueness of the measures $\nu_{t^{u}}, \nu_{t^{s}}$ in equation (26) imply that, if there exists a measure of full dimension, then it already coincides with $\nu_{t^{u}}$ and $\nu_{t^{s}}$. Thus, we have the following result.

Corollary 3.5. Assume $m$ is a measure of full dimension for $g$. Then $m=\nu_{t^{u}}=$ $\nu_{t^{s}}$. In particular, there exists at most one measure of full dimension.

\section{Measures of Full Dimension}

In this section we identify the conditions under which a measure of full dimension exists. We start with the volume-preserving case.

Theorem 4.1. Let $g$ be volume-preserving. Then $t^{u}=t^{s}$, and $\nu_{t^{u}}$ is a measure of full dimension for $g$.

Proof. We have $|a|=1$. Therefore Proposition 3.1 implies that $P^{u}=P^{s}$. Thus, Theorem 3.4 yields $t^{u}=t^{s}$. Therefore, by equations (9) and (27), we conclude that $\operatorname{dim}_{H}\left(\nu_{t^{u}}\right)=\operatorname{dim}_{H} J$, which implies that $\nu_{t^{u}}$ is a measure of full dimension.

Remark. As noted in the introduction, in the volume-preserving case, the existence of a measure of full dimension was already shown by Friedland and Ochs [FO]. They proved that the existence of a measure of full dimension follows from the fact that $\left|\operatorname{det} D g^{n}(p)\right|=1$ holds for every periodic point $p$ with period $n$. They also observed that in this case the measure of full dimension is equivalent to the $t$-dimensional Hausdorff measure, where $t$ is the Hausdorff dimension of $J$.

We now consider the volume-decreasing case. Let $\mathcal{S}$ denote the set of all saddle points of $g$. Note that $J=\overline{\mathcal{S}}$, see [BS1]. For $p \in \mathcal{S}$ with period $n(p)$ we denote by $\lambda^{u / s}(p)$ the eigenvalues of $D g^{n(p)}(p)$, where $\left|\lambda^{s}(p)\right|<1<\left|\lambda^{u}(p)\right|$. In the next theorem we provide equivalent conditions for the existence of a measure of full dimension.

Theorem 4.2. Assume $g$ is volume-decreasing. Then the following are equivalent.

i) $g$ admits a measure of full dimension.

ii) The unstable pressure function $P^{u}$ is affine.

iii) The stable pressure function $P^{s}$ is affine.

iv) The measure of maximal entropy is a measure of full dimension for $g$. 
v) The quantity

$$
\left|\lambda^{u}(p)\right|^{\frac{1}{n(p)}}
$$

is independent of the periodic point $p \in \mathcal{S}$.

Proof. $i i) \Leftrightarrow$ iii) follows from Proposition 3.1.

i) $\Rightarrow i$ i) Let us assume that $g$ admits a measure of full dimension. It is shown in [Wo2] that $t^{s}<t^{u}$. Corollary 3.5] implies that $\nu_{t^{u}}=\nu_{t^{s}}$ is the measure of full dimension. So $\Lambda\left(t^{u}\right)=\Lambda\left(t^{s}\right)$, and this in turn implies by Proposition 3.3 that the function $\Lambda(t)$ is constant. Therefore, equation (19) implies that $P^{u}$ is affine.

$i i)+i i i) \Rightarrow i v)$ Recall that $\nu_{0}$ is the unique measure of maximal entropy for $g$, see Section 3. The topological entropy of $\left.g\right|_{J}$ is equal to $\log d$ (see [BS3]). Thus $P^{u}(0)=P^{s}(0)=\log d$. Equation 19 and Proposition 3.1 imply

$$
\begin{aligned}
& \frac{d P^{u}}{d t}(0)=-\Lambda(0), \\
& \frac{d P^{s}}{d t}(0)=-\Lambda(0)+\log |a| .
\end{aligned}
$$

Since $P^{u}$ and $P^{s}$ are affine, Theorem 3.4 and equation (26) imply

$$
\operatorname{dim}_{H} J=\frac{\log d}{\Lambda(0)}+\frac{\log d}{\Lambda(0)-\log |a|} .
$$

But by Young's formula (9), the right-hand side of (31) is equal to $\operatorname{dim}_{H}\left(\nu_{0}\right)$. Thus, $\nu_{0}$ is a measure of full dimension.

$i v) \Rightarrow v$ ) If $\nu_{0}$ is a measure of full dimension for $g$, then, by Corollary 3.5, we have $\nu_{0}=v_{t^{u}}$. Moreover, it follows from Theorem 3.4 that $t^{u}>0$. Therefore, $v$ ) follows from Proposition 4.5 of [B].

$v) \Rightarrow i i$ ) follows again from Proposition 4.5 of $[\mathrm{B}$.

Finally, $i v) \Rightarrow i$ ) is trivial.

Remark. As mentioned in the introduction, it is very likely that for volume-decreasing maps a measure of full dimension never exists. This can be seen by using property $v$ ) of Theorem 4.2, because if such a map $g$ exists, then the parameter defining $g$ provides solutions of a countable infinite set of algebraic equations, which is indeed a very strong conclusion.

Let us assume that a volume-decreasing map $g$ admits a measure of full dimension. Then it follows by equation (28) that $g$ belongs to a real codimension one algebraic subset of parameter space. Using a perturbation argument, it is not too hard to see that we can find arbitrarily close to $g$ a map $g^{\prime}$ for which (28) does not hold; in particular, $g^{\prime}$ has no measure of full dimension. Here we mean close with respect to the topology on hyperbolic parameter space induced by the parameter of the mapping (see [Wo1] for details). On the other hand, if $g$ admits no measure of full dimension, then (28) does not hold for $g$. It can be easily shown that there is a neighborhood of $g$ such that for each map $g^{\prime}$ in this neighborhood (28) does not hold. Therefore, there exists a dense open subset of parameters admitting no measure of full dimension. We leave the details to the reader. 


\section{Measures of Maximal Dimension}

In this section we establish the existence of a measure of maximal dimension.

We first consider the volume-preserving case. In this situation it follows from Corollary 3.5 and Theorem 4.1 that $\nu_{t^{u}}$ is the unique measure of full dimension for $g$. By definition, every measure of full dimension is also a measure of maximal dimension. Therefore, we obtain the following.

Theorem 5.1. Assume $g$ is volume-preserving. Then $\nu_{t^{u}}$ is the unique measure of maximal dimension for $g$.

We now consider the volume-decreasing case. The following theorem is the main result of this paper.

Theorem 5.2. Assume $g$ is volume-decreasing. Then there exists a measure of maximal dimension for $g$. If $m$ is a measure of maximal dimension for $g$, then there exists $t^{s}<t<t^{u}$ such that $m$ is the equilibrium measure of the potential $-t \phi^{u}$, that is, $m=\nu_{t}$.

Proof. Since $g$ is volume-decreasing, we have $t^{s}<t^{u}$ (see Wo2]).

We first assume that $g$ admits a measure of full dimension. In this case application of Theorem 4.2 and Proposition 4.5 of $[\mathrm{B}]$ implies that $\nu_{t}$ does not depend on $t$. Moreover, $\nu_{t}$ is the unique measure of full and therefore also of maximal dimension. Thus, the theorem holds.

We now assume that $f$ has no measure of full dimension. Thus, by Theorem 4.2 the functions $P^{u / s}$ are not affine.

Assertion 1. There exists $\epsilon>0$ such that $\Delta$ is strictly increasing on $\left[0, t^{s}+\epsilon\right)$ and strictly decreasing on $\left(t^{u}-\epsilon, \infty\right)$.

Proof of Assertion 1. Theorem 3.4 and the fact that $P^{u / s}$ are strictly decreasing functions [property ii) of the topological pressure] imply that $P^{s}(t)>0$ for all $t \in\left[0, t^{s}\right)$. Analogously we have $P^{u}(t)>0$ for all $t \in\left[0, t^{u}\right)$. We conclude from Proposition 3.3 equation (24) and an elementary continuity argument that there exists $\epsilon>0$ such that

$$
\frac{d \Delta}{d t} \geq 0
$$

in $\left[0, t^{s}+\epsilon\right)$, and all zeros of the derivative of $\Delta$ in $\left[0, t^{s}+\epsilon\right)$ are isolated. Therefore, $\Delta$ is strictly increasing in $\left[0, t^{s}+\epsilon\right)$. A similar argument shows that there exists $\epsilon>0$ such that $\Delta$ is strictly decreasing in $\left(t^{u}-\epsilon, \infty\right)$.

Assertion 1 implies that there exists $t^{*} \in\left[t^{s}+\epsilon, t^{u}-\epsilon\right]$ such that

$$
\operatorname{dim}_{H}\left(\nu_{t^{*}}\right)=\sup _{t \geq 0} \Delta(t) .
$$

Assertion 2. The measure $\nu_{t^{*}}$ is a measure of maximal dimension.

Proof of Assertion 2. Let $\left(m_{k}\right)_{k \in \mathbf{N}}$ be a sequence in $M\left(J,\left.g\right|_{J}\right)$ such that

$$
\lim _{k \rightarrow \infty} \operatorname{dim}_{H}\left(m_{k}\right)=\delta(g) .
$$

By Assertion 1, we may assume, without loss of generality, that $\operatorname{dim}_{H}\left(\nu_{0}\right)=\Delta(0)<$ $\operatorname{dim}_{H}\left(m_{k}\right)$ for all $k \in \mathbf{N}$. Recall that $\nu_{0}$ is the unique measure of maximal entropy 
of $g$ (see Section 3). We now may conclude by Young's formula (9) that

$$
\Lambda\left(\nu_{0}\right)>\Lambda\left(m_{k}\right)
$$

for all $k \in \mathbf{N}$. Again by Assertion 1, we may assume, without loss of generality, that $\operatorname{dim}_{H}\left(\nu_{t^{u}}\right)=\Delta\left(t^{u}\right)<\operatorname{dim}_{H}\left(m_{k}\right)$ for all $k \in \mathbf{N}$. Equation (26) implies that

$$
\frac{h_{m_{k}}(g)}{\Lambda\left(m_{k}\right)}<\frac{h\left(t^{u}\right)}{\Lambda\left(t^{u}\right)}
$$

for all $k \in \mathbf{N}$. Therefore, Young's formula (9) implies that

$$
\frac{h_{m_{k}}(g)}{\Lambda\left(m_{k}\right)-\log |a|}>\frac{h\left(t^{u}\right)}{\Lambda\left(t^{u}\right)-\log |a|}
$$

for all $k \in \mathbf{N}$. Equations (36) and (37) imply that $h_{m_{k}}(g)>h\left(t^{u}\right)$, and therefore again by equation (36) we obtain

$$
\Lambda\left(m_{k}\right)>\Lambda\left(t^{u}\right)
$$

for all $k \in \mathbf{N}$. Since $\Lambda$ is continuous, equations (35) and (38) imply that for all $k \in \mathbf{N}$ there exists $t_{k} \in\left(0, t^{u}\right)$ such that

$$
\Lambda\left(m_{k}\right)=\Lambda\left(t_{k}\right) .
$$

Thus, the variational principle (10) implies that

$$
h_{m_{k}}(g) \leq h\left(t_{k}\right)
$$

hence

$$
\operatorname{dim}_{H}\left(m_{k}\right) \leq \Delta\left(t_{k}\right)
$$

for all $k \in \mathbf{N}$. This implies that

$$
\operatorname{dim}_{H}\left(m_{k}\right) \leq \operatorname{dim}_{H}\left(\nu_{t^{*}}\right)
$$

for all $k \in \mathbf{N}$. We conclude that $\nu_{t^{*}}$ is a measure of maximal dimension.

To complete the proof of the theorem we have to show the following.

Assertion 3. For every measure $m$ of maximal dimension there exists $t^{s}<t<t^{u}$ such that $m$ is the equilibrium measure of the potential $-t \phi^{u}$.

Proof of Assertion 3. Let $m$ be a measure of maximal dimension. We apply to $m$ (instead of $m_{k}$ ) the same argument as in the proof of Assertion 2. This implies that there exists $t \in\left(0, t^{u}\right)$ such that $\Lambda(m)=\Lambda(t)$. Since $\operatorname{dim}_{H}(m) \geq \Delta(t)$, we may deduce by equation (9) that $h_{m}(g) \geq h(t)$. On the other hand, since $\nu_{t}$ is the equilibrium measure of the potential $-t \phi^{u}$, we may conclude by (10) and (11) that $h_{m}(g) \leq h(t)$. Hence $h_{m}(g)=h(t)$. Therefore, the uniqueness of the equilibrium measure of the potential $-t \phi^{u}$ implies that $m=\nu_{t}$. Finally, Assertion 1 implies that $t \in\left(t^{s}, t^{u}\right)$. This completes the proof.

Remarks. The following heuristic argument was given by McCluskey and Manning [MM to state the existence of a measure of maximal dimension in the case of $C^{2}$ axiom A diffeomorphisms of real surfaces. Since the entropy map is upper semicontinuous, it can be shown that the map $\nu \mapsto \operatorname{dim}_{H}(\nu)$, defined on the set of all ergodic invariant measures, is also upper semi-continuous. It is now suggested in [MM] that this implies the existence of a measure of maximal dimension. To make this argument rigorous we need to show that there exists a sequence of ergodic invariant measures $m_{k}$ with $\operatorname{dim}_{H}\left(m_{k}\right) \rightarrow \delta(g)$ having an ergodic weak* limit. 
Whether this holds is not clear since the set of all ergodic invariant measures is dense in the set of all invariant measures with respect to the weak* topology; see Proposition 21.9 in [DGS]. In particular, the set of all ergodic invariant measures is not closed.

In the following we describe properties of the measures of maximal dimension.

Corollary 5.3. Assume that $g$ admits no measure of full dimension. Let $t \geq 0$ be such that $\nu_{t}$ is a measure of maximal dimension. Then

$$
\delta(g)=2 t+\frac{P^{u}(t) \log |a|}{\Lambda(t)^{2}} .
$$

Proof. By Proposition 3.3. equation (24) and Theorem [5.2, a necessary condition for $\nu_{t}$ being a measure of maximal dimension is

$$
P^{u}(t)(\Lambda(t)-\log |a|)^{2}+\left(P^{u}(t)+t \log |a|\right) \Lambda(t)^{2}=0 .
$$

Therefore, the result follows from equation (23).

Corollary 5.4. The set of all measures of maximal dimension is finite.

Proof. Assume first that $g$ admits a measure of full dimension. Then, this measure is the unique measure of maximal dimension. If $g$ has no measure of full dimension, then the function $\Delta$ is a non-constant real analytic function on $[0, \infty)$. Therefore, it follows from the uniqueness theorem for real analytic functions that $\Delta$ has only finitely many maxima in $\left[t^{s}, t^{u}\right]$. The result follows from Theorem $[5.2$,

Corollary 5.5. Every measure $\nu$ of maximal dimension is Bernoulli.

Proof. Since $\left.g\right|_{J}$ is topological mixing (see [BS1]), the result follows from the fact that $\nu$ is an equilibrium measure of a Hölder continuous potential (see [B], Thm. $4.1)$.

\section{Dependence on Parameters}

Let $A$ denote an open subset of $\mathbb{C}^{k}$ and let $\left(g_{\lambda}\right)_{\lambda \in A}$ be a holomorphic family of hyperbolic polynomial automorphisms of $\mathbb{C}^{2}$ of fixed dynamical degree $d>1$.

We denote by $J_{\lambda}$ the Julia set, by $a_{\lambda}$ the Jacobian determinant, and by $P_{\lambda}^{u / s}$ the unstable/stable pressure functions of $g_{\lambda}$. We also write $\Delta_{\lambda}(t)$ instead of $\Delta(t)$. First, we show that $\delta(g)$ depends continuously on the parameter of the mapping.

Theorem 6.1. The function $\lambda \mapsto \delta\left(g_{\lambda}\right)$ is continuous in $A$.

Proof. Let $\lambda_{0} \in A$. The result of [VW] implies that there exist $\epsilon>0$ and a real analytic function

$$
\mathcal{P}: B\left(\lambda_{0}, \epsilon\right) \times[0, \infty) \rightarrow \mathbf{R},
$$

such that $\mathcal{P}(\lambda,)=.P_{\lambda}^{u}$ for all $\lambda \in B\left(\lambda_{0}, \epsilon\right)$. Therefore, equations (19) and (23) imply that

$$
\mathcal{D}: B\left(\lambda_{0}, \epsilon\right) \times[0, \infty) \rightarrow \mathbf{R}, \quad(\lambda, t) \mapsto \Delta_{\lambda}(t)
$$

is also a real analytic function. Now we may conclude by Theorem 5.1 and Theorem 5.2 that

$$
\delta\left(g_{\lambda}\right)=\max _{t \in[0,2]} \mathcal{D}(\lambda, t) .
$$

The result follows by an elementary continuity argument. 
Remark. McCluskey and Manning [MM] considered $C^{2}$ Axiom A diffeomorphisms of real surfaces. They showed that for these mappings $\delta(g)$ depends continuously on the mapping with respect to the $C^{2}$ topology.

Finally, we show that $\delta(g)$ depends plurisubharmonically on the parameter of the mapping.

Theorem 6.2. The function $\lambda \mapsto \delta\left(g_{\lambda}\right)$ is plurisubharmonic in $A$.

Proof. Let $g_{0} \in A$ and let $L$ be a complex line in $\mathbb{C}^{k}$ containing $g_{0}$. Then there exists a holomorphic family $\left(g_{\lambda}\right)_{\lambda \in D}$, where $D$ is a disk with center 0 in $\mathbb{C}$ such that $\left\{g_{\lambda}: \lambda \in D\right\}$ is a neighborhood of $g_{0}$ in $L \cap A$. If the radius of $D$ is small enough, then there exists a family $\left(\kappa_{\lambda}\right)_{\lambda \in D}$, where each $\kappa_{\lambda}$ is the uniquely defined conjugacy between $\left.g_{0}\right|_{J_{0}}$ and $\left.g_{\lambda}\right|_{J_{\lambda}}$. Therefore, $T_{\lambda}=\left(\kappa_{\lambda}\right)_{*}$ defines a family of bijections from $M\left(J_{0},\left.g_{0}\right|_{J_{0}}\right)$ to $M\left(J_{\lambda},\left.g_{\lambda}\right|_{J_{\lambda}}\right)$. Moreover, we have $h_{\nu}\left(g_{0}\right)=h_{T_{\lambda}(\nu)}\left(g_{\lambda}\right)$ for all $\nu \in M\left(J_{0},\left.g_{0}\right|_{J_{0}}\right)$ and all $\lambda \in D$ (see Wo1 for the details). In [Wo1] we showed that if $\nu \in M\left(J_{0},\left.g_{0}\right|_{J_{0}}\right)$ is fixed, then $\lambda \mapsto \Lambda\left(T_{\lambda}(\nu)\right)$ is a harmonic function in $D$. We conclude by Young's formula (9) that

$$
\delta\left(g_{\lambda}\right)=\sup _{\nu \in M\left(J_{0}, g_{0} \mid J_{0}\right)}\left(\frac{h_{\nu}\left(g_{0}\right)}{\Lambda\left(T_{\lambda}(\nu)\right)}+\frac{h_{\nu}\left(g_{0}\right)}{\Lambda\left(T_{\lambda}(\nu)\right)-\log \left|a_{\lambda}\right|}\right) .
$$

The functions $\lambda \mapsto \Lambda\left(T_{\lambda}(\nu)\right), \lambda \mapsto \Lambda\left(T_{\lambda}(\nu)\right)-\log \left|a_{\lambda}\right|$ are harmonic in $D$. Note that $x \mapsto x^{-1}$ is a convex function on $\mathbf{R}^{+}$. This implies that the functions $\lambda \mapsto$ $\Lambda\left(T_{\lambda}(\nu)\right)^{-1}, \lambda \mapsto\left(\Lambda\left(T_{\lambda}(\nu)\right)-\log \left|a_{\lambda}\right|\right)^{-1}$ are subharmonic in $D$. The continuous function $\lambda \mapsto \delta\left(g_{\lambda}\right)$ is therefore given by the supremum over a family of subharmonic functions. We conclude that the function $\lambda \mapsto \delta\left(g_{\lambda}\right)$ is subharmonic in $D$. This completes the proof.

\section{ACKNOWLEDGEMENT}

I would like to thank Eric Bedford and Marlies Gerber for many useful discussions and comments during the preparation of this paper. I also would like to thank the referee for his detailed report, and for suggesting various improvements. This paper was written while I was visiting Indiana University and I would like to thank the Department of Mathematics for its warm hospitality.

\section{REFERENCES}

[B] R. Bowen, Equilibrium states and the ergodic theory of Anosov diffeomorphisms, Lecture Notes in Math. 470, Springer-Verlag, Berlin, 1975 MR 56:1364

[BS1] E. Bedford and J. Smillie, Polynomial diffeomorphisms of $\mathbb{C}^{2}$ : Currents, equilibrium measure and hyperbolicity, Invent. Math. 103 (1991), 69 - 99 MR 92a:32035

[BS2] E. Bedford and J. Smillie, Polynomial diffeomorphisms of $\mathbb{C}^{2} 2$ : Stable manifolds and recurrence, J. Amer. Math. Soc. 4 (1991), 657 - 679 MR 92m:32048

[BS3] E. Bedford and J. Smillie, Polynomial diffeomorphisms of $\mathbb{C}^{2} 3$ : Ergodicity, exponents and entropy of the equilibrium measure, Math. Ann. 294 (1992), 395 - 420 MR 93k:32062

[BW1] L. Barreira and C. Wolf, Pointwise dimension and ergodic decompositions, preprint, 2002

[BW2] L. Barreira and C. Wolf, Measures of maximal dimension for hyperbolic diffeomorphisms, Comm. Math. Phys., to appear

[DGS] M. Denker, C. Grillenberger and K. Sigmund, Ergodic theory on compact spaces, Lecture Notes in Math. 527, Springer-Verlag, Berlin, 1976 MR 56:15879

[DU] M. Denker and M. Urbanski, On Sullivan's conformal measures for rational maps on the Riemann sphere, Nonlinearity 4 (1991), 365-384 MR 92f:58097

[FM] S. Friedland and J. Milnor, Dynamical properties of plane polynomial automorphisms, Ergodic Theory and Dynam. Syst. 9 (1989), 67-99 MR 90f:58163 
[FO] S. Friedland and G. Ochs, Hausdorff dimension, strong hyperbolicity and complex dynamics, Discrete and Continuous Dynam. Syst. 4 (1998), 405 - 430 MR 99g:58091

$[\mathrm{KH}]$ A. Katok and B. Hasselblatt, Introduction to the modern theory of dynamical systems, Cambridge University Press, 1995 MR 96c:58055

[MM] H. McCluskey and A. Manning, Hausdorff dimension for horseshoes, Ergodic Theory and Dynam. Syst. 3 (1983), 251 - 260 MR 85j:58127

[R] D. Ruelle, Thermodynamic formalism. The mathematical structures of classical equilibrium statistical mechanics, Addison-Wesley, Reading, MA, 1978 MR 80g:82017

[VW] A. Verjovsky and H. Wu, Hausdorff dimension of Julia sets of complex Hénon mappings, Ergodic Theory and Dynam. Syst. 16 (1996), 849 - 861 MR 97g:58143

[Wa] P. Walters, An introduction to ergodic theory, Graduate Texts in Mathematics, vol. 79, Springer, 1982 MR 84e:28017

[Wo1] C. Wolf, Dimension of Julia sets of polynomial automorphisms of $\mathbb{C}^{2}$, Michigan Mathematical Journal 47 (2000), 585 - 600 MR 2002a:37072

[Wo2] C. Wolf, Hausdorff and topological dimension for polynomial automorphisms of $\mathbb{C}^{2}$, Ergodic Theory and Dynam. Syst. 22 (2002), 1313-1327

[Y] L.-S. Young, Dimension, entropy and Lyapunov exponents, Ergodic Theory and Dynam. Syst. 2 (1982), 109-124 MR 84h:58087

Department of Mathematics, Wichita State University, Wichita, Kansas 67260-0033

E-mail address: cwolf@math.twsu.edu

URL: http://www.math.wichita.edu/ ${ }^{\sim}$ cwolf/ 